\title{
BMJ Open Assessment of medium-term cardiovascular disease risk after Japan's 2011 Fukushima Daiichi nuclear accident: a retrospective analysis
}

\author{
Haruka Toda, ${ }^{1}$ Shuhei Nomura, ${ }^{1}$ Stuart Gilmour, ${ }^{1}$ Masaharu Tsubokura, ${ }^{2}$ \\ Tomoyoshi Oikawa, ${ }^{3}$ Kiwon Lee, ${ }^{4}$ Grace Y Kiyabu, ${ }^{5}$ Kenji Shibuya ${ }^{1}$
}

To cite: Toda H, Nomura S, Gilmour S, et al. Assessment of medium-term cardiovascular disease risk after Japan's 2011 Fukushima Daiichi nuclear accident: a retrospective analysis. BMJ Open 2017;7:e018502. doi:10.1136/ bmjopen-2017-018502

- Prepublication history and additional material for this paper are available online. To view these files, please visit the journal online (http://dx.doi org/10.1136/bmjopen-2017018502).

Received 11 July 2017 Revised 25 October 2017 Accepted 22 November 2017

\section{CrossMark}

${ }^{1}$ Department of Global Health Policy, Graduate School of Medicine, The University of Tokyo, Tokyo, Japan ${ }^{2}$ Department of Radiation Protection, Minamisoma Municipal General Hospital, Minamisoma, Japan ${ }^{3}$ Department of Neurosurgery, Minamisoma Municipal General Hospital, Minamisoma, Japan ${ }^{4}$ International Center for AIDS Care and Treatment Program (ICAP), Columbia University, New York City, New York, USA ${ }^{5}$ Health Economics and Outcomes Research, CreativCeutical K.K, Tokyo, Japan

Correspondence to Ms Haruka Toda; haruka-toda@umin.ac.jp

\section{ABSTRACT}

Objective To assess the medium-term indirect impact of the 2011 Fukushima Daiichi nuclear accident on cardiovascular disease (CVD) risks and to identify whether risk factors for CVD changed after the accident.

Participants Residents aged 40 years and over participating in annual public health check-ups from 2009 to 2012, administered by Minamisoma city, located about 10 to $40 \mathrm{~km}$ from the Fukushima Daiichi nuclear plant. Methods The sex-specific Framingham CVD risk score was considered as the outcome measure and was compared before (2009-2010) and after the accident (2011-2012). A multivariate regression analysis was employed to evaluate risk factors for CVD.

Results Data from 563 individuals (60.2\% women) aged 40 to 74 years who participated in the check-ups throughout the study period was analysed. After adjusting for covariates, no statistically significant change was identified in the CVD risk score postaccident in both sexes, which may suggest no obvious medium-term health impact of the Fukushima nuclear accident on CVD risk. The risk factors for CVD and their magnitude and direction (positive/negative) did not change after the accident. Conclusions There was no obvious increase in CVD risks in Minamisoma city, which may indicate successful management of health risks associated with CVD in the study sample.

\section{INTRODUCTION}

On 11 March 2011, Japan was struck by the Great East Japan Earthquake and subsequent tsunami. ${ }^{1}$ A magnitude 9.0 earthquake off the Pacific coast of Japan triggered a massive tsunami that destroyed thousands of houses and killed 18877 people. $^{2}$ As of September 2016, five and a half years following the tsunami, over 2500 people are still missing and presumed dead. ${ }^{3}$ This tsunami also caused a nuclear accident at the Fukushima Daiichi nuclear power plant, located on the Pacific coast of Fukushima Prefecture (figure 1). Coupled with the massive earthquake and tsunami, the post-Fukushima
Strengths and limitations of this study

- This is the first study to assess medium-term cardiovascular disease (CVD) risk factors after the Fukushima accident.

- Using validated CVD risk assessment tools, the study provides a consistent estimate of how risk changed over time.

- By providing a longitudinal assessment of a cohort of individuals who attended health checks consistently, the study was able to eliminate bias due to changing populations being assessed and to follow the impact of the disaster on individuals.

- The health check-ups were undertaken on a voluntary basis and only by those aged $40-74$ in Minamisoma city, potentially limiting generalisability to a wider population.

nuclear accident evacuations disrupted more than 164000 lives in Fukushima. ${ }^{4}$ Within 1 week of the accident, $8 \%$ of the total population (two million) of Fukushima had to move either elsewhere within Fukushima or out of the prefecture. ${ }^{5}$ The United Nations Scientific Committee on the Effects of Atomic Radiation and WHO have concluded that the predicted risk of lifetime cancer after the Fukushima nuclear accident is very low in the general population in Japan. ${ }^{67}$ Only infants and children who were most exposed in Fukushima may have a higher risk based on model calculations. ${ }^{78}$ In contrast to the likely low risk of radiation-related health consequences, ${ }^{78}$ non-radiological health effects postaccident have been reported; the disaster may have had other medium-term and longterm health impacts, including elevated markers of metabolic risk, ${ }^{9-11}$ increases in diabetes and hyperlipidaemia ${ }^{12}$ and higher prevalence of psychological distress. ${ }^{13}$

There is growing evidence that major disasters contribute to an increase in 


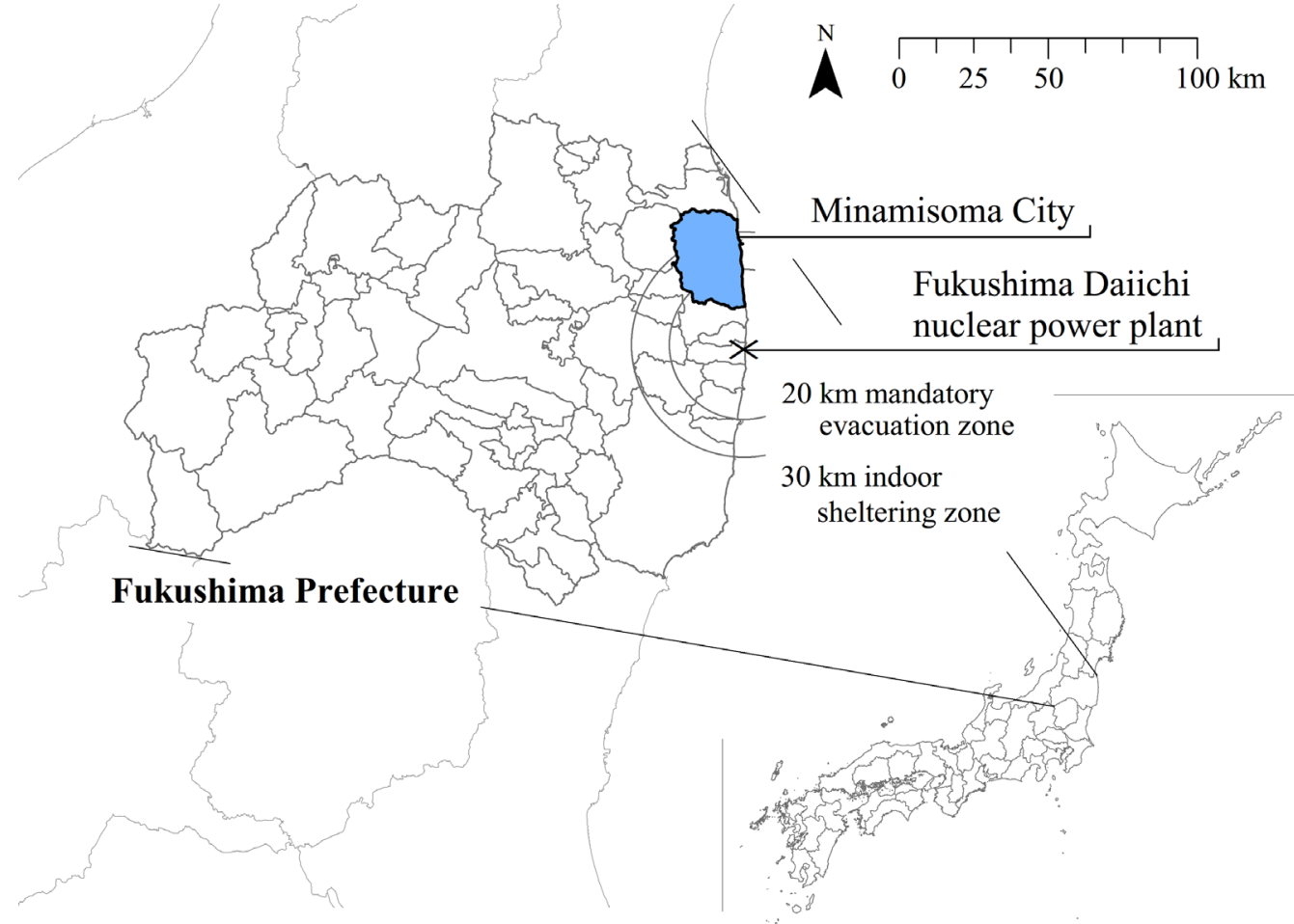

Figure 1 Map of Fukushima Prefecture and location of Fukushima Daiichi nuclear power plant and Minamisoma city

cardiovascular disease (CVD) risks, including increased serum total cholesterol, blood pressure, fasting insulin, reduced high-density lipoprotein (HDL) cholesterol and/ or smoking behaviour and high psychological stress. ${ }^{14-18}$ These elevated risks are found in the immediate aftermath of disasters and may last over the medium term. Previous studies reported a threefold increase in acute myocardial infarction-related hospital admission rates 2-3years after the 2005 Hurricane Katrina in the USA. ${ }^{19} 20$ Gilmour et al found a medium-term increase in CVD risk in the areas most affected by the 2011 Fukushima nuclear accident. ${ }^{21}$ This increase may have been due to anxiety/ concern about radiation exposure, ${ }^{13}$ reduced outdoor activity levels resulting in reduced physical exercise ${ }^{22}$ and reluctance to access healthcare. ${ }^{12}$

Understanding the direct and indirect health risks in the postacute periods of disasters could help identify entry points for action and strategic directions for health policy and practice on medium-term to longterm disaster risk management. While the short-term effects of disaster on CVD risk have been well evaluated, ${ }^{14-162023}$ information on the effects of disaster on CVD risk in the postacute periods is relatively scarce. The aim of this study was to provide understanding of the risk factors associated with identified medium-term increases in CVD incidents following the Fukushima Daiichi nuclear accident by studying health data on a sample of the local population most at risk of increased CVD-related health outcomes. This is the first study to assess medium-term CVD risk factors after the Fukushima accident.

\section{METHODS}

Design, settings and participants

This study was conducted in Minamisoma city, located $10-40 \mathrm{~km}$ from the Fukushima Daiichi nuclear power plant (figure 1). The city contains both mandatory and voluntary evacuation zones, which were lifted on 31 May 2016. A total of 636 people in Minamisoma city died due to the tsunami, ${ }^{24}$ accounting for $39.7 \%$ of the total tsunami deaths $(\mathrm{n}=1604)$ in Fukushima. ${ }^{3}$ As of July 2012, 388 deaths were identified as 'disaster-related deaths, ${ }^{25}$ a term officially defined by the Reconstruction Agency as deaths due to indirect damage caused by the earthquake, tsunami and nuclear accident. ${ }^{26}$ The majority of the disaster-related deaths are attributed to the evacuation of the elderly following the accident. ${ }^{27} 28$ The population of Minamisoma city was about 71500 before the accident and decreased to 66800 in March 20121 year after the accident. ${ }^{29-31}$ As of August 2016, 2412 Minamisoma residents were still living in temporary housing. ${ }^{32}$

In the present study, we used data from public health check-up records from 2009 to 2012 administered by the Minamisoma city office to compare CVD risks before (2009-2010) and after the accident (2011-2012). The public health check-ups consist of clinical tests and surveys conducted to screen and assess overall health conditions. They are performed annually by all municipalities in Japan at designated community centres and medical institutions during June to August. The checks are free for all people aged 40 to 74 and covered by the National Health Insurance programme, which is designed for citizens who are not insured by any employment-based health 
insurance programme (ie, unemployed and self-employed, including farmers and fishermen). A health check-up notification is sent to each household every year based on the municipality's family registry and also announced in the city's public relations magazine.

The health check-up data used in this study included a physical examination, blood sample and self-reported medical history and lifestyle survey. The selfreported medical history survey asked participants about family history of heart disease, hypertension, diabetes and hyperlipidaemia; use of hypertension, diabetes or hyperlipidaemia medicines and present treatment status for some specific diseases based on a four-option scaleno disease, receiving outpatient treatment, recovered or left untreated. The lifestyle survey asked about habits related to alcohol, smoking and lifestyle, including exercise, walking speed and eating speed. More details of the medical history and lifestyle survey can be found in online supplementary tables 1 and 2, respectively. Because the public health check-up is voluntary, individuals do not necessarily attend the health check-up every year. Each participant is given a personal identification number by the city office which was used in this study to track subject data across the study period. In this study, we considered only those who had received health check-ups every year from 2009 to 2012. By doing so, we could follow the study participants' demographic and clinical characteristics during the study period. Some individuals in 2012 took the check-ups several times in that year, and we used only the first check-up for these subjects.

\section{CVD risk score}

We used the sex-specific Framingham CVD risk point scale as the primary outcome. This scale was developed and adopted from the Framingham Heart Study-a longterm, ongoing cardiovascular cohort study on residents of the town of Framingham, Massachusetts in the USA. ${ }^{33}$ The CVD risk point scale is generalisable to other populations and widely used globally in clinical practice to evaluate and compare individuals' risks for developing a CVD event. $^{34-39}$ It incorporates age, HDL cholesterol level, total cholesterol level, systolic blood pressure (SBP), use of medicines for hypertension, smoking status and fasting glucose level. $^{33}$

Originally, the CVD risk point calculation used subject's age ${ }^{33}$ but in this study we did not take age into account for the point calculation. This study exclusively considered only those who participated in the health check-ups for all 4years from 2009 to 2012, which indicates that during the study period the age distribution of the study participants all moved upwards by 1 year for each year. Therefore, if age was considered in the CVD risk point calculation, the age-attributed CVD risk point would also increase with year, and it would be difficult to distinguish the effects of time and age on the CVD risk point score in the analyses.

Online supplementary tables 3 and 4 present the CVD risk calculation table for men and women, respectively.
In these tables, the five risk factors-HDL, total cholesterol level, SBP with/without use of medicines for hypertension, smoking status, diabetic status-were scored using the method adopted by D'Agostino et al. ${ }^{33}$ Then, each participant at each time point (2009, 2010, 2011 and 2012) in the study was assigned a CVD risk point by summing up the scores of these five factors.

In this study, the clinical characteristics data used for the point calculation were extracted from the blood sample test (for HDL and SBP) and self-reported medical history and lifestyle survey (for use of medicines for hypertension and smoking status). Ideally, the total cholesterol level should also be extracted from the blood sample test; however, it was not measured in the public health check-ups. Therefore, we employed the self-reported medical history survey to estimate total cholesterol level for the CVD point calculation in the following manner: those who were receiving outpatient treatment for hyperlipidaemia or left untreated were assumed to have total cholesterol levels of over $200 \mathrm{mg} / \mathrm{dL}$; and others were assumed to have levels less than $200 \mathrm{mg} / \mathrm{dL}$. Diabetic status (no/yes) was also based on the self-reported medical history survey. Those who were receiving outpatient treatment for diabetes or left untreated were considered diabetic.

\section{Data analysis}

Comparison of CVD risk point before and after the accident

To assess the medium-term impact of Japan's Fukushima Daiichi nuclear accident on CVD risk, we compared the change in the CVD risk point after the accident with preaccident values, for men and women separately, adjusting for age. Preaccident baseline risk point was defined as the average CVD risk point in 2009 and 2010. Statistical significance of the change postaccident was examined using a paired t-test.

\section{Risk factor analysis}

To identify if and how risk factors for CVD changed after the accident, we employed multivariate linear regression. The regression models were constructed for men and women, separately. Independent variables included elements of the physical examination, blood sample tests, self-reported medical history and lifestyle survey.

We also considered year as an independent variable and its interaction terms with other independent variables to examine postaccident changes of the relationship between outcome variable (CVD risk point) and independent variables, such as the magnitude and direction (positive/negative) of the effect of the independent variables on the CVD risk score. Because HDL, total cholesterol level, SBP, use of medicines for hypertension, smoking status and diabetic status were used as input in calculating CVD risk points, these variables were not included in the regression models to avoid possible overadjustment. ${ }^{40}$

Since all the participants had four data points during the study period (2009-2012), the regression model included a random effect at individual level. Model selection 
was performed using backward-stepwise selection with a $\mathrm{P}>0.05$ to remove, starting with all possible variables, until significant variables were left. Variables that were well-known or suspected risk factors, for example, family history of heart disease, were incorporated into the final model regardless of their statistical significance. All statistical analyses were conducted using STATA/MP V.14. In this study, $\mathrm{P}$ values less than 0.05 were reported as statistically significant.

\section{RESULTS}

\section{Characteristics of study participants}

A total of 7855 individuals participated in at least one or more health check-ups during the study period from 2009 to 2012. After excluding those who did not take the check-ups throughout the 4-year study period (7292 individuals, $92.8 \%$ ), 563 individuals ( $60.2 \%$ women) were used in the present study. This limited sample size was partially due to the fact that only 1145 individuals took the check-up in 2011 (the year of the Fukushima accident), which was performed only months after the accident, while the other years had more participants (5272 in 2009, 5042 in 2010 and 4122 in 2012). Online supplementary table 5 shows the comparison between included and excluded participants. The excluded participants had statistically significant differences to the included participants, but from a clinical perspective, all results were within the normal range among the excluded participants (eg, the normal triglyceride level was less than $150 \mathrm{mg} / \mathrm{dL}$ ). There were no clinically important, statistically significant differences in several demographic and clinical characteristics in baseline years (2009-2010). Given that even those clinical characteristics that differed in the excluded group remained well within the normal range for all measurements, it is unlikely that these small differences between the groups have a noticeable effect on our findings.

Table 1 reports both demographic and clinical characteristics of the study participants. The preaccident baseline data (2009-2010) was compared with the postaccident data (2011-2012), separately, using a paired t-test. The mean age of men was 64.3 years in 2009 (SD: 5.9). Women had a mean age of 62.6 years in 2009 (SD: 6.1).

Online supplementary tables 1 and 2 present the results of self-reported medical history and the lifestyle survey, respectively. Because all variables from the medical history and lifestyle survey were binary or categorical, the data from 2010 were compared to the postaccident data of 2011 and 2012, separately, using $\chi^{2}$ test or Fisher's exact, and showed little change in specific components of the CVD risk score. As shown in online supplementary table 1, there were no significant differences in lifestyle habits such as smoking and alcohol before and after the accident.

\section{Comparison of CVD risk score before and after the accident}

The mean CVD risk score for baseline (2009 and 2010) was 1.36 (SD: 3.06) for men and 0.19 (SD: 3.19) for women.
No statistically significant differences were observed between before and after the accident in CVD risk point after adjusting for age. This means that there might be no medium-term impact of the Japan's Fukushima Daiichi nuclear accident on CVD risk in both sexes.

\section{Risk factor analyses}

Table 2 shows the results of multivariate linear regression analysis for risk factors of CVD risk score. Models were separately constructed for men and women. For both sexes, final models included year, age, BMI, family history of heart disease, use of medicines for diabetes and hyperlipidaemia and alcohol consumption. After adjusting for covariates, age was significantly associated only among women. A 1-year increase in age was associated with only a 0.09 -point increase in CVD risk point only (95\% CI 0.05 to 0.12 ). Each $1 \mathrm{~kg} / \mathrm{m}^{2}$ increase in BMI was significantly associated with a 0.23 -point (95\% CI 0.14 to 0.33 ) and 0.30 -point (95\% CI 0.23 to 0.36 ) increase in the CVD risk point in men and women, respectively.

Other binary variables, such as family history of heart disease and use of medicines for diabetes and hyperlipidaemia also had a significant positive correlation with the CVD risk score in both sexes. Furthermore, year was not significantly associated with change in score in either sex after adjusting for covariates, suggesting no medium-term impact of the Fukushima nuclear accident on CVD risk. Importantly, there was no significant interaction between independent variables and year, and thus no interaction terms were incorporated into the final models. This means that the risk factors for CVD and their magnitude and direction of influence (positive/negative) might not have changed after the accident.

Given the limited sample size (partially due to the limited number of health check-up participation in 2011), we also conducted sensitivity analysis to validate the regression models. In the sensitivity analysis, we included participants of the check-ups from 2009, 2010 and 2012, which increased the sample size from 563 to 1916 individuals, representing $5.9 \%$ of the total population in the age group of 40 to 74 years in the city postaccident. Online supplementary table 6 presents the results of the sensitivity analysis for the regression models, which produced similar results to the original regression models, suggesting limited systematic bias.

\section{DISCUSSION}

This study compared the Framingham CVD risk score before and after the 2011 Fukushima Daiichi nuclear accident and evaluated if and how risk factors for CVD changed after the accident. There was no statistically significant change in the CVD risk score postaccident in either sex after adjusting for covariates, suggesting no obvious medium-term impact of the Fukushima nuclear accident on CVD risk. The risk factors for CVD and their magnitude and direction (positive/negative) did not change after the accident. 


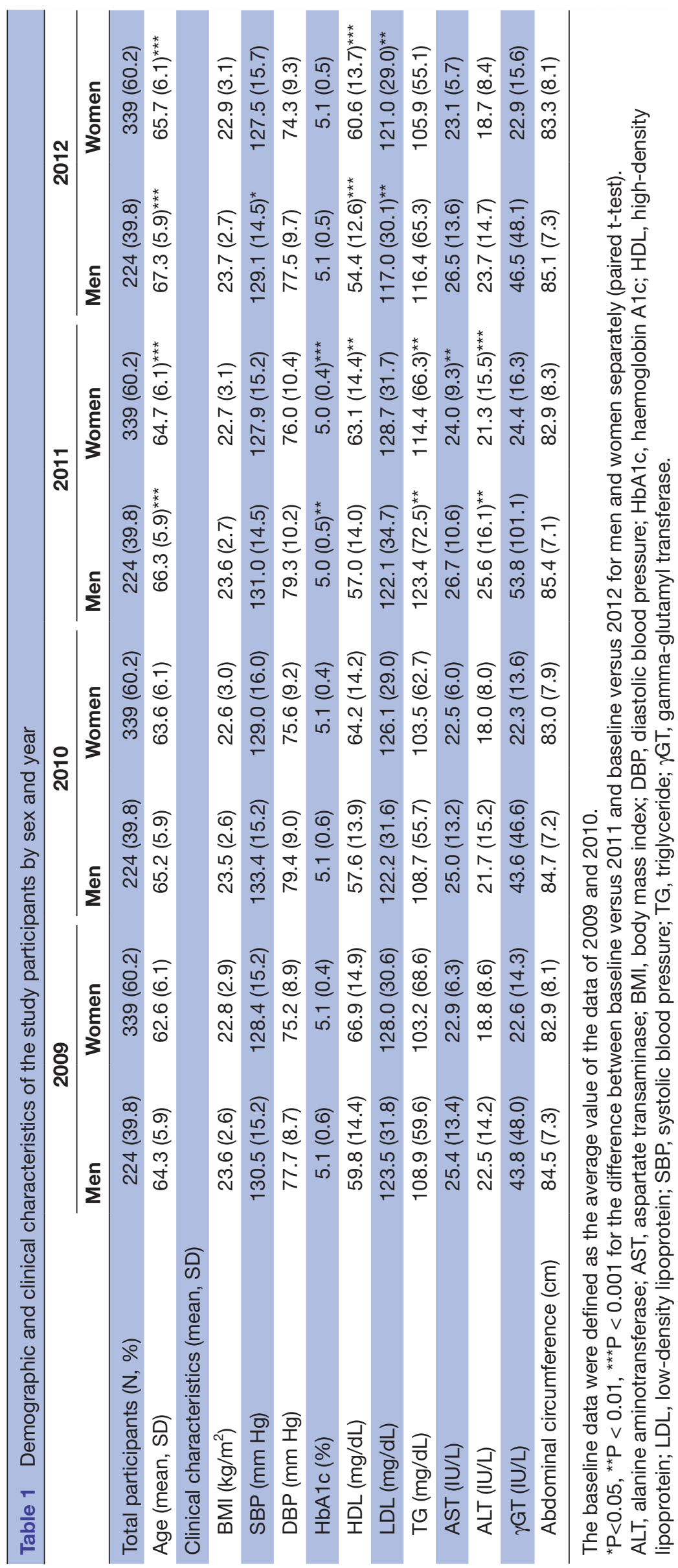


Table 2 Regression analysis for CVD risk point for men and women

\begin{tabular}{|c|c|c|c|c|c|c|}
\hline \multirow[b]{2}{*}{ Variable } & \multicolumn{3}{|c|}{ Men $(n=224)$} & \multicolumn{3}{|c|}{ Women $(n=339)$} \\
\hline & Coefficient & $95 \% \mathrm{Cl}$ & $P$ value & Coefficient & $95 \% \mathrm{Cl}$ & $P$ value \\
\hline \multicolumn{7}{|l|}{ Year } \\
\hline Baseline $^{*}$ & Reference & & & Reference & & \\
\hline 2011 & -0.12 & -0.37 to 0.12 & 0.33 & -0.02 & -0.25 to 0.20 & 0.83 \\
\hline 2012 & -0.27 & -0.57 to 0.03 & 0.08 & 0.09 & -0.17 to 0.34 & 0.52 \\
\hline Age & 0.04 & -0.01 to 0.09 & 0.10 & 0.09 & 0.05 to 0.12 & $<0.001$ \\
\hline BMI & 0.23 & 0.14 to 0.33 & $<0.001$ & 0.30 & 0.23 to 0.36 & $<0.001$ \\
\hline \multicolumn{7}{|c|}{ Family history of heart disease } \\
\hline No & Reference & & & Reference & & \\
\hline Yes & 0.75 & 0.23 to 1.26 & $<0.01$ & 0.34 & 0.02 to 0.66 & $<0.05$ \\
\hline \multicolumn{7}{|l|}{ Use of medicines } \\
\hline \multicolumn{7}{|l|}{ Diabetes } \\
\hline No & Reference & & & Reference & & \\
\hline Yes & 4.24 & 3.47 to 5.00 & $<0.001$ & 6.36 & 5.29 to 7.44 & $<0.001$ \\
\hline \multicolumn{7}{|c|}{ Hyperlipidaemia } \\
\hline No & Reference & & & Reference & & \\
\hline Yes & 1.73 & 1.27 to 2.19 & $<0.001$ & 1.98 & 1.61 to 2.35 & $<0.001$ \\
\hline \multicolumn{7}{|c|}{ Alcohol consumption } \\
\hline None & Reference & & & Reference & & \\
\hline Rarely & 0.20 & -0.41 to 0.80 & 0.53 & -0.06 & -0.39 to 0.27 & 0.72 \\
\hline Sometimes & 0.06 & -0.45 to 0.56 & 0.83 & 0.11 & -0.36 to 0.58 & 0.65 \\
\hline Everyday & 0.36 & -0.20 to 0.93 & 0.21 & 0.29 & -0.39 to 0.10 & 0.41 \\
\hline
\end{tabular}

*Baseline indicates the average CVD risk point of 2009 and 2010.

$\mathrm{BMI}$, body mass index; CVD, cardiovascular disease.

Blood pressure-a key element of the Framingham CVD risk point calculation-is a very important, wellknown risk factor for an array of cardiovascular and related diseases. ${ }^{41}$ Past studies provided evidence that a disaster's impact on blood pressure may persist in the postacute phase. ${ }^{42}{ }^{43}$ However, we did not observe a significant increase in blood pressure 2 years after the Fukushima accident in Minamisoma city (table 1). This is consistent with findings from a previous study. ${ }^{12}$ These facts may indicate successful disaster risk management of chronic health risks (particularly of high blood pressure) in Minamisoma city.

As a response to the sociodemographic changes in the population due to the Fukushima nuclear accident, ${ }^{27} 2844$ Minamisoma city has started to implement a set of unique strategic policy reforms with the aim of creating a resilient and healthy population in the city in the following areas: health promotion, disease prevention, dental health, radiation monitoring and education and food and nutrition education. ${ }^{45}$ This policy reform gives high priority to primary prevention and advocates early detection, awareness, prevention and treatment of hypertension and its consequences. This study found that the proportion of those who used medicines for hypertension increased in Minamisoma city from $35.7 \%$ and $28.3 \%$ in 2010 to
$44.2 \%$ and $36.6 \%$ in 2012 for men and women, respectively, and suggests that the Minamisoma's strategic policy may have had some impact in improving management of hypertension and associated risks.

Although the findings of this study may suggest no medium-term change in CVD risk factors in Minamisoma city after the Fukushima accident, there are aspects that should be carefully considered to fully understand CVD risk after the accident. First, Ohira $e t a t^{46}$ found a significant medium-term blood pressure increase in their prefecture-level longitudinal study after the accident, while our study and Nomura $e t a l^{2}$ revealed no significance increase in Minamisoma city. Given this, the findings from our study with limited sample size should be carefully interpreted. However, Ohira's study was prefecture wide, while our study was focused on those closest to and most affected by the disaster, and our study may be more relevant to postdisaster recovery planning in heavily affected communities.

Nomura $e t a l^{12}$ showed that the prevalence of diabetes and hyperlipidaemia in Minamisoma city significantly increased 2 years after the accident (2013 and 2014) by up to $60 \%$ and $30 \%$ in comparison with the preaccident years, respectively, while no increase was observed in 2011 and 2012 for both diseases. It is known that diabetes and 
hyperlipidaemia are relatively more difficult than hypertension to control after a disaster. Since blood glucose and cholesterol monitoring require invasive procedures, self-monitoring is less accepted by patients, resulting in reduced patient compliance in diabetes and hyperlipidaemia monitoring and treatment. ${ }^{12} 47$ The regression analysis in our study showed that use of medicines for diabetes and hyperlipidaemia was significantly associated with CVD risk (table 2). These results may indicate the possibility that CVD risk could also increase a couple of years after the accident (ie, postmedium phase), triggered through diabetes-related and hyperlipidaemiarelated cardiovascular risk factors, such as increased total increased cholesterol and fasting insulin. Therefore, disease-specific measures could be necessary to aid the management of potential CVD risk, so that disaster resilience to CVD will be further strengthened in the long term.

\section{Limitations}

This study has several limitations. First, the Framingham CVD risk score outcome was originally developed based on the Framingham Heart Study, in which 99\% of cohort participants were Caucasian. ${ }^{33}$ Therefore, this risk point is not necessarily generalisable for other populations. Another Japanese-specific CVD risk point score such as NIPPON DATA $80 / 90$ or Suita Study score could have been used, but NIPPON DATA 80/90 does not include HDL and use of medicines for hypertension, and the Suita Study required estimation of glomerular filtration rate, which was not possible in Minamisoma. However, some past studies have applied the Framingham CVD risk score to the Japanese population with reasonable accuracy; therefore, it may be acceptable in Minamisoma. ${ }^{48-50}$ In addition, originally the CVD risk point calculation used total cholesterol level, but we did not take exact total cholesterol level from the health check-up data, because the data only included HDL and low-density lipoprotein.

Second, the health check-ups were undertaken on a voluntary basis and only offered to those aged 40 to 74 insured under the National Health Insurance programme, potentially biasing the results and limiting our ability to generalise to a wider population.

Third, it is well known that postdisaster evacuation may engender differential vulnerability in the population, particularly elderly people, in terms of chronic health conditions, but we were not able to consider evacuation experience in the analysis as no such data were available. However, although values of some clinical characteristics have been shown to be significantly associated with evacuation experience in Minamisoma city in the postacute phase, ${ }^{12}$ the magnitude of this association was very small, and the influence of this limitation on our findings is likely to be very small.

Finally, our study was conducted only on those who were present in Minamisoma for both pre-earthquake years and both postearthquake years, raising the possibility of selection bias. We tested this by comparing included and excluded individuals on key clinical characteristics and by conducting sensitivity analysis with a larger sample based on people who attended three rather than all four health check-ups. Both of these tests found no evidence of any systemic bias that might affect the study, and the replication of the results with a larger, more inclusive sample suggests that such bias is minimal and our findings are likely to be reflective of real trends in risk factors in this population.

\section{CONCLUSIONS}

No statistically significant change was identified in CVD risk postaccident in either sex, after adjusting for covariates, indicating no obvious medium-term impact of the Fukushima nuclear accident on CVD risk. These results might suggest successful management of health risks associated with CVD in Minamisoma city. Promotion of disease-specific and target-specific measures can make an important contribution to further strengthen local disaster to protect people from CVD.

Acknowledgements We extend our appreciation to the Minamisoma city employees and Minamisoma Municipal General Hospital staff for data collection and management. Without their involvement, our study could not have reached its present form.

Contributors HT, SN and SG designed the study and contributed to writing the manuscript. MT and TO contributed to planning the health screening program and SG, MT, TO, KL and GYK performed data collection. HT, SN and SG performed statistical analysis and drafted the article. KS contributed to scientific review. All authors read and approved the final manuscript.

\section{Competing interests None declared.}

Ethics approval Ethical approval for the study was granted by the ethics committee of Minamisoma Municipal General Hospital, Japan, authorisation number 28-02.

Provenance and peer review Not commissioned; externally peer reviewed. Data sharing statement № additional data are available.

Open Access This is an Open Access article distributed in accordance with the Creative Commons Attribution Non Commercial (CC BY-NC 4.0) license, which permits others to distribute, remix, adapt, build upon this work non-commercially, and license their derivative works on different terms, provided the original work is properly cited and the use is non-commercial. See: http://creativecommons.org/ licenses/by-nc/4.0/

C Article author(s) (or their employer(s) unless otherwise stated in the text of the article) 2017. All rights reserved. No commercial use is permitted unless otherwise expressly granted.

\section{REFERENCES}

1. Minamisoma Municipal Office, Reconstruction Planning Depertment Risk Management Division. Disaster record in Minamisoma City (In Japanese). 2016. http://www.city.minamisoma.Ig.jp/index.cfm/10, 15930,c,html/15930/20160608-092612.pdf (accessed 25 Dec 2016).

2. Ministry of Health, Labour and Welfare of Japan. List of vital statistical surveys conducted by Ministry of Health Labour, and Welfare of Japan (In Japanese) Tokyo. 2011. http://www.mhlw.go.jp/ toukei/saikin/hw/jinkou/kakutei11/dl/14_x34.pdf (accessed 25 Dec 2016).

3. National Police Agency of Japan, Emergency Disaster Countermeasures Headquarters. Damage situation and police counter measures associated with 2011 Tohoku district - off the Pacific Ocean Earthquake (In Japanese) Tokyo. 2016. http://www. pref.fukushima.lg.jp/site/portal/list281-1040.html (accessed 25 Dec 2016). 
4. Fukushima Prefectural Government. The population decrease after the disaster in Fukushima. 2016. http://www.pref.fukushima.lg.jp/ site/portal/list281-1040.html (accessed 25 Dec 2016).

5. Fukushima Prefectural Government. The trend of evacuation in Fukushima. 2016. http://www.pref.fukushima.lg.jp/site/portal/list271. html (accessed 25 Dec 2016).

6. United Nations Scientific Committee on the Effects of Atomic Radiation. United Nations Scientific Committee on the Effects of Atomic Radiation UNSCEAR 2013 Report to the General Assembly, with scientific annexes. New York: United Nations Scientific Committee on the Effects of Atomic Radiation, 2013.

7. World Health Organization. Health risk assessment from the nuclear accident after the 2011 Great East Japan Earthquake and tsunami, based on a preliminary dose estimation. Geneva: World Health Organization, 2013.

8. United Nations Scientific Committee on the Effects of Atomic Radiation. Annex A: Levels and effects of radiation exposure due to the nuclear accident after the 2011 Great East-Japan Earthquake and tsunami. New York: United Nations Scientific Committee on the Effects of Atomic Radiation, 2014.

9. Hashimoto S, Nagai M, Fukuma S, et al. Influence of post-disaster evacuation on incidence of metabolic syndrome. $J$ Atheroscler Thromb 2017;24:327-37.

10. Satoh $\mathrm{H}$, Ohira T, Hosoya $\mathrm{M}$, et al. Evacuation after the Fukushima Daiichi nuclear power plant accident is a cause of diabetes: results from the Fukushima Health Management Survey. J Diabetes Res 2015;2015:1-9.

11. Tsubokura M, Takita M, Matsumura T, et al. Changes in metabolic profiles after the Great East Japan Earthquake: a retrospective observational study. BMC Public Health 2013;13:267-67.

12. Nomura S, Blangiardo M, Tsubokura M, et al. Postnuclear disaster evacuation and chronic health in adults in Fukushima, Japan: a longterm retrospective analysis. BMJ Open 2016;6:e010080.

13. Yabe $\mathrm{H}$, Suzuki $\mathrm{Y}$, Mashiko $\mathrm{H}$, et al. Psychological distress after the Great East Japan Earthquake and Fukushima Daiichi Nuclear Power Plant accident: results of a mental health and lifestyle survey through the Fukushima Health Management Survey in FY2011 and FY2012. Fukushima J Med Sci 2014;60:57-67.

14. Aoki T, Fukumoto $Y$, Yasuda $S$, et al. The Great East Japan Earthquake Disaster and cardiovascular diseases. Eur Heart $J$ 2012;33:2796-803.

15. Ogawa K, Tsuji I, Shiono K, et al. Increased acute myocardial infarction mortality following the 1995 Great Hanshin-Awaji earthquake in Japan. Int J Epidemiol 2000;29:449-55.

16. Omama S, Yoshida Y, Ogasawara K, et al. Influence of the great East Japan earthquake and Tsunami 2011 on occurrence of cerebrovascular diseases in Iwate, Japan. Stroke 2013;44:1518-24.

17. World Health Organization. Avoiding heart attacks and strokes don't be a victim - protect yourself. Geneva: World Health Organization, 2005.

18. Zhang XQ, Chen $M$, Yang Q, et al. Effect of the Wenchuan earthquake in China on hemodynamically unstable ventricular tachyarrhythmia in hospitalized patients. Am J Cardiol 2009;103:994-7.

19. Gautam S, Menachem J, Srivastav SK, et al. Effect of Hurricane Katrina on the incidence of acute coronary syndrome at a primary angioplasty center in New Orleans. Disaster Med Public Health Prep 2009;3:144-50.

20. Jiao Z, Kakoulides SV, Moscona J, et al. Effect of Hurricane Katrina on incidence of acute myocardial infarction in New Orleans three years after the storm. Am J Cardiol 2012:109:502-5.

21. Gilmour S, Sugimoto A, Nomura S, et al. Long-term changes in stroke-related hospital admissions after the Fukushima triple disaster. J Am Geriatr Soc 2015;63:2425-6.

22. Nagai M, Ohira T, Yasumura S, et al. Association between evacuation condition and habitual physical activity in Great East Japan Earthquake evacuees: The Fukushima Health Management Survey. Nihon Koshu Eisei Zasshi 2016;63:3-10.

23. Leor J, Poole WK, Kloner RA. Sudden cardiac death triggered by an earthquake. N Engl J Med Overseas Ed 1996;334:413-9.

24. Minamisoma City Office. The disater records of Minamisoma City (In Japanese). 2013. http://www.city.minamisoma.lg.jp/index.cfm/10, 15930,144,html (accessed 25 Dec 2016).

25. Minamisoma City Office. Causes of disaster-related deaths in the Minamisoma City and countermeasures (In Japanese). http://www. reconstruction.go.jp/topics/2-9.teisyutusiryou.pdf (accessed $25 \mathrm{Dec}$ 2016).

26. Reconstruction Agency the Comittee of Disaster-Related Death. The report of disaster-related death in the Great East Japan Earthquake. 2012. http://www.reconstruction.go.jp/topics/240821_ higashinihondaishinsainiokerushinsaikanrenshinikansuruhoukoku.pdf (accessed 25 Dec 2016)

27. Nomura S, Gilmour S, Tsubokura M, et al. Mortality risk amongst nursing home residents evacuated after the Fukushima nuclear accident: a retrospective cohort study. PLoS One 2013;8:e60192.

28. Nomura S, Blangiardo M, Tsubokura M, et al. Post-nuclear disaster evacuation and survival amongst elderly people in Fukushima: a comparative analysis between evacuees and non-evacuees. Prev Med 2016;82:77-82.

29. Minamisoma City Office. Basic resident register: population by distinction and households on Feburary 2012 (In Japanese). 2012. http://www.city.minamisoma.lg.jp/index.cfm/8,4704,c,html/4704/ ooazabetuH240229.pdf (accessed 25 Dec 2016).

30. Minamisoma City Office. Basic resident register: Population by distinction and households on Feburary 2011 (In Japanese). 2011. http://www.city.minamisoma.lg.jp/index.cfm/8,4704,c,html/4704/ ooazabetuH230228.pdf (accessed 25 Dec 2016).

31. Statistics Bureau, Ministry of Internal Affairs and Communications, Statistics Japan. Population cencus at Fukushima in 2010. 2011. http://www.e-stat.go.jp/SG1/estat/List.do?bid=000001033715\& cycode $=0$ (accessed 25 Dec 2016).

32. Minamisoma City Office. The situation of evacuation and city residents (In Japanese). 2016. https://www.city.minamisoma.lg.jp/ index.cfm/10,853,58,html (accessed 25 Dec 2016).

33. D'Agostino RB, Vasan RS, Pencina MJ, et al. General cardiovascular risk profile for use in primary care: the Framingham Heart Study. Circulation 2008;117:743-53.

34. Joffres M, Falaschetti E, Gillespie C, et al. Hypertension prevalence, awareness, treatment and control in national surveys from England, the USA and Canada, and correlation with stroke and ischaemic heart disease mortality: a cross-sectional study. BMJ Open 2013;3:e003423.

35. National Institutes of Health, Department of Health and Human Services. Estimate of 10-year risk for coronary heart disease Framingham point scores Maryland. 2016. https://www.nhlbi.nih. gov/health-pro/guidelines/current/cholesterol-guidelines/quick-deskreference-html/10-year-risk-framingham-table (accessed $25 \mathrm{Dec}$ 2016).

36. Framingham Heart Study. Cardiovascular disease (10-year risk) Maryland. 2016. https://www.framinghamheartstudy.org/riskfunctions/cardiovascular-disease/10-year-risk.php (accessed $25 \mathrm{Dec}$ 2016).

37. Lyngbakken MN, Skranes JB, de Lemos JA, et al. Impact of smoking on circulating cardiac troponin i concentrations and cardiovascular events in the general population. The HUNT Study 2016;134:1962-72.

38. Peng $\mathrm{H}$, Jiao $\mathrm{Y}$, Zeng $\mathrm{Q}$, et al. Utility of Framingham general cardiovascular disease risk score for predicting 10-year cardiovascular risk in an Inner Mongolian population: a prospective cohort study. Int J Cardiol 2014;172:274-5

39. Canadian Cardiovascular Society. Calculators and forms, Framingham risk score worksheet 2013. http://www.ccs.ca/images/ Guidelines/Tools_and_Calculators_En/Dyslipidmia_Guidelines_Tool_ 2013 Grey.pdf

40. Szklo M, Nieto FJ. Epidemiology: beyond the basics. 3rd edn Burlington: Jones and Barlett Learning, 2007:227-98.

41. Mancia G, De Backer G, Dominiczak A, et al. 2007 ESH-ESC Practice guidelines for the management of arterial hypertension: ESH-ESC Task force on the management of arterial hypertension. $J$ Hypertens 2007;25:1751-62.

42. Konno S, Munakata M. Blood pressure elevation lasting longer than 1 year among public employees after the Great East Japan Earthquake: The Watari Study. Am J Hypertens 2017;30:120-3.

43. Giorgini P, Striuli R, Petrarca M, et al. Long-term blood pressure changes induced by the 2009 L'Aquila earthquake: assessment by $24 \mathrm{~h}$ ambulatory monitoring. Hypertens Res 2013;36:795-8.

44. Leppold C, Tsubokura M, Ozaki A, et al. Sociodemographic patterning of long-term diabetes mellitus control following Japan's 3.11 triple disaster: a retrospective cohort study. BMJ Open 2016;6:e011455

45. Minamisoma City Office. Comprehensive plan of health and welfare in Minamisoma City (In Japanese. 2013. http://www.city. minamisoma.lg.jp/index.cfm/8,12563,c,html/12563/20130619131157.pdf (accessed 25 Dec 2016).

46. Ohira T, Hosoya M, Yasumura S, et al. Evacuation and risk of hypertension after the Great East Japan earthquake: the Fukushima health management survey. Hypertension 2016;68:558-64.

47. Frontino G, Meschi F, Bonfanti R, et al. Future perspectives in glucose monitoring sensors. Eur Endocrinol 2010;9:21-6. 
48. Suka M, Sugimori H, Yoshida K. Application of the updated Framingham risk score to Japanese men. Hypertens Res 2001;24:685-9.

49. Zhu B, Haruyama $Y$, Muto $T$, et al. Evaluation of a community intervention program in Japan using Framingham risk score and estimated 10-year coronary heart disease risk as outcome variables: a non-randomized controlled trial. BMC Public Health 2013;13:219-19.

50. Nishimura K, Okamura T, Watanabe M, et al. Predicting coronary heart disease using risk factor categories for a Japanese urban

population, and comparison with the framingham risk score: the suita study. J Atheroscler Thromb 2014;21:784-98. 